\title{
Corticosteroid transdermal delivery significantly improves arthritis pain and functional disability
}

\author{
Tommaso Iannitti ${ }^{1,2}$ • Michael F. McDermott ${ }^{3}$ - Carmen Laurino ${ }^{2,4}$ - Andrea Malagoli ${ }^{5}$. \\ Beniamino Palmieri ${ }^{2,4}$
}

Published online: 7 December 2016

(C) The Author(s) 2016. This article is published with open access at Springerlink.com

\begin{abstract}
Arthritis is characterized by pain and functional limitation affecting the patients' quality of life. We performed a clinical study to investigate the efficacy of a betamethasone valerate medicated plaster (Betesil) in improving pain and functional disability in patients with arthritis and osteoarthritis. We enrolled 104 patients affected by osteoarthritis $(n=40)$ or arthritis $(n=64)$ in different joints. Patients received diclofenac sodium cream ( $2 \mathrm{~g}$, four times a day) or a 2.25-mg dose of Betesil applied to the painful joint every night before bedtime for 10 days. Pain and functional disability were assessed, by the Visual Analogue Scale (VAS) and Western Ontario McMaster Universities Osteoarthritis Index (WOMAC) scores. Redness was assessed by clinical inspection, and edema by the "fovea sign" method. C-reactive protein (CRP) was also measured; CRP can be used to cost-effectively monitor the pharmacological treatment efficacy and is
\end{abstract}

Tommaso Iannitti

tommaso.iannitti@gmail.com

1 KWS BioTest, Marine View Office Park, Portishead, Somerset BS20 7AW, UK

2 Poliambulatorio del Secondo Parere, 41100 Modena, Italy

3 National Institute for Health Research - Leeds Musculoskeletal Biomedical Research Unit (NIHR-LMBRU) and Leeds Institute of Rheumatic and Musculoskeletal Medicine (LIRMM), Wellcome Trust Brenner Building, St James's University Hospital, Beckett Street, Leeds, West Yorkshire LS9 7TF, UK

4 Department of General Surgery and Surgical Specialties, University of Modena and Reggio Emilia Medical School, Surgical Clinic, 41100 Modena, Italy

5 Department of Medical and Surgical Sciences for Children and Adults, University of Modena and Reggio Emilia, 41100 Modena, Italy increased during the acute-phase response, returning to physiological values after tissue recovery and functional restoration. All measurements were at baseline and at 10-day follow-up. At 10-day follow-up, a greater improvement in VAS and WOMAC pain and WOMAC stiffness and functional limitation scores from baseline was observed in patients treated with Betesil compared with diclofenac (all $p<0.01$ ). At 10-day follow-up, improvement in redness, edema, and CRP levels from baseline was also greater in patients treated with Betesil compared with diclofenac (all $p<0.01$ ). This study demonstrates the safety and efficacy of transdermal delivery of betamethasone valerate in patients affected by arthritis and osteoarthritis.

Keywords Betamethasone valerate · Transdermal drug delivery $\cdot$ Pain $\cdot$ Functional disability $\cdot$ Stiffness $\cdot$ C-reactive protein $\cdot$ Arthritis $\cdot$ Osteoarthritis

\section{Introduction}

Arthritis is a pathological condition that causes pain and inflammation in a joint. Osteoarthritis $(\mathrm{OA})$ is the most common form of arthritis and is defined as a degenerative mesenchymal disease affecting an estimated $10 \%$ of the world's population over 60 years $[1,2]$. Symptoms include pain, stiffness, and functional limitation, leading to loss of autonomy and poor quality of life [3]. Various treatment options are available for OA management. These include (1) non-steroidal anti-inflammatory drugs (NSAIDs) for pain management [4], (2) bisphosphonates to decrease pain and improve functionality by preserving the structural integrity of subchondral bone [5], (3) pulsed electromagnetic field therapy [6], and (4) viscosupplementation, with hyaluronic acid alone or in combination with bisphosphonates or NSAIDs, to improve pain 
and functional activity [7-9]. Indeed, viscosupplementation with hyaluronic acid improves articular cartilage degeneration and decreases osteophyte formation, as shown by experimental studies using OA models [10, 11]. Topical corticosteroids also decrease pain and improve joint functionality [12, 13]. The non-pharmacological management of OA includes education and self-management, exercise and weight loss, assistive devices, alternative and complementary approaches, and surgical interventions [14].

Transdermal delivery of betamethasone valerate for treatment of arthritis So far, only a few studies have investigated the clinical efficacy of transdermal delivery of corticosteroids for OA management. Corticosteroids for the treatment of arthritis may be administered by iontophoresis, a non-invasive technique that allows transdermal drug delivery. Betamethasone valerate (BMV) is a synthetic, moderately active corticosteroid without mineralocorticoid properties, [15] which binds the intracellular cytoplasmic glucocorticoid receptor and translocates it to the nucleus to function as a ligand-activated transcription factor [16]. Eight sessions of dexamethasone iontophoresis have been used for treatment of juvenile idiopathic arthritis affecting the temporomandibular joint [17]. Resolution of pain occurred in $73 \%$ of the patients who had pain at baseline. On the other hand, a second study, evaluating the efficacy of a transdermal steroid delivery vs. placebo by iontophoresis or phonophoresis for the treatment of patients with trapeziometacarpal arthritis, did not show significant differences between treatments [18].

The aim of this study was to compare the efficacy of transdermal BMV and diclofenac sodium cream in patients affected by arthritis and OA, in order to determine the best therapeutic option in terms of pain, redness, edema, and functional disability.

\section{Materials and methods}

This study was performed at the Poliambulatorio del Secondo Parere clinic (Modena, Italy) in accordance with the Declaration of Helsinki and approved by the Institutional Review Board at Poliambulatorio del Secondo Parere (Modena, Italy). All patients signed the informed consent and agreed to data collection and review.

Patients' demographics and disease characteristics A total of 104 patients (62 males and 42 females) participated in this study. Originally, 106 patients were recruited but two patients, affected by hip arthritis, dropped out soon after recruitment due to the development of a symptomatic herniated disc and excluded from data analysis. Inclusion criteria were symptomatic arthritis and OA with a Visual
Analogue Scale (VAS) pain score $\geq 60 \mathrm{~mm}$. Exclusion criteria were hypersensitivity or allergy to the active component of the plaster, presence of skin edema, and joint effusions. Patients had a mean age of $57.3 \pm 1.09$ [mean \pm standard error of the mean (SEM)]. Patients were diagnosed on the basis of medical examination and orthopedic evaluation using ultrasound, radiography, and magnetic resonance imaging as follows: bilateral knee OA $(n=24)$, hip arthritis $(n=4)$, OA with bulging disc between L4 and L5 $(n=16)$, sciatic nerve inflammation deriving from spinal arthritis occurring at L4, L5, and S1 $(n=20)$, shoulder arthritis $(n=28)$, and carpal tunnel syndrome associated with arthritis affecting the wrist $(n=12)$ (Table 1$)$. All patients signed the informed consent.

Study design Patients were matched on the basis of age, sex, and type of pathology $(p=0.958, p=0.318$, and $p=0.84$, respectively; Table 1). Patients were instructed to apply a 2.250-mg BMV medicated plaster (Betesil; $n=52$ ) on the painful joint every night before bedtime for 10 days or diclofenac sodium cream $(n=52 ; 2 \mathrm{~g})$ on the painful joint four times a day for 10 days. If pain relief was not adequate, patients were instructed to take diclofenac sodium orally as rescue medication ( $50 \mathrm{mg}$, up to three capsules per day).

Pain and functional disability assessment VAS (0-100 mm; $0 \mathrm{~mm}=$ minimum pain $; 100 \mathrm{~mm}=$ maximum pain $)$ and Western Ontario McMaster Universities Osteoarthritis Index (WOMAC) scores were used to evaluate the efficacy of Betesil on pain and functional disability at 10-day follow-up. WOMAC is based on five items related to pain (subscore 0 $20 ; 0=$ minimum pain; $20=$ maximum pain), two to stiffness (subscore $0-8 ; 0=$ minimum stiffness; $8=$ maximum stiffness), and 17 to functional limitation (subscore $0-68 ; 0=$ minimum functional limitation; 68 = maximum functional limitation).

Redness and edema assessment Redness was assessed by visual clinical inspection (subscore $0-3 ; 0=$ absence of redness, 1 = slight redness, 2 = moderate redness, and 3 = intense redness). Edema was assessed by the "fovea sign" method [19]. The fovea sign is positive when there is exquisite tenderness compared with the contralateral side and is scored as follows: subscore $0-3,0=$ absence of edema, $1=2-\mathrm{mm}$ edema following depression of the skin, $2=4-\mathrm{mm}$ edema following depression of the skin, and $3=$ 5-mm edema following depression of the skin.

C-reactive protein The venous blood of all fasting subjects was drawn in the morning, and C-reactive protein (CRP) levels were detected by immunoturbidimetric method, using an automated analyzer. CRP has been 
Table 1 Patients' demographics and disease characteristics at baseline and 10-day follow-up

\begin{tabular}{|c|c|c|c|}
\hline & Diclofenac sodium cream $(n=52)$ & Betesil $(n=52)$ & $p$ \\
\hline Age & $57.06 \pm 1.52$ & $57.54 \pm 1.6$ & 0.958 \\
\hline $\begin{array}{l}\text { Female patients } \\
\text { Male patients }\end{array}$ & $\begin{array}{l}18(35 \%) \\
34(65 \%)\end{array}$ & $\begin{array}{l}24(46 \%) \\
28(54 \%)\end{array}$ & 0.318 \\
\hline $\begin{array}{l}\text { Arthritis (non-rheumatoid) } \\
\text { Osteoarthritis }\end{array}$ & $\begin{array}{l}33(63 \%) \\
19(37 \%)\end{array}$ & $\begin{array}{l}31(60 \%) \\
21(40 \%)\end{array}$ & 0.84 \\
\hline $\begin{array}{l}\text { Carpal tunnel syndrome/wrist arthritis } \\
\text { Hip arthritis }\end{array}$ & $\begin{array}{l}7(13 \%) \\
2(4 \%)\end{array}$ & $\begin{array}{l}5(10 \%) \\
2(4 \%)\end{array}$ & 0.902 \\
\hline Knee osteoarthritis & $10(19 \%)$ & $14(27 \%)$ & \\
\hline Osteoarthritis with bulging disk (L4-L5) & $9(17 \%)$ & $7(13 \%)$ & \\
\hline Sciatic nerve inflammation/spinal arthritis & $9(17 \%)$ & $11(21 \%)$ & \\
\hline Shoulder arthritis & $15(29 \%)$ & $13(25 \%)$ & \\
\hline VAS (mm; baseline) & $75(70-80)$ & $87(78-91.25)$ & $<0.01$ \\
\hline VAS (mm; follow-up) & $65(60-70)$ & $35(32-43.25)$ & $<0.01$ \\
\hline WOMAC (pain; baseline) & $14(10-17)$ & $15(10-19)$ & 0.384 \\
\hline WOMAC (pain; follow-up) & $12(9-16)$ & $7(4-10)$ & $<0.01$ \\
\hline WOMAC (functional limitation; baseline) & $51.5(40.75-60)$ & $51(39-61.25)$ & 0.706 \\
\hline WOMAC (functional limitation; follow-up) & $47(38.5-55)$ & $25.5(17-39.25)$ & $<0.01$ \\
\hline WOMAC (stiffness; baseline) & $5(4-7)$ & $6(4.75-8)$ & 0.083 \\
\hline WOMAC (stiffness; follow-up) & $4.5(3-6)$ & $3(2-4.25)$ & $<0.01$ \\
\hline CRP (mg/l; baseline) & $4.25(2.98-6.03)$ & $4.75(3-6)$ & 0.848 \\
\hline CRP (mg/l; follow-up) & $3.65(2.1-5.73)$ & $2.25(1-3)$ & $<0.01$ \\
\hline $\begin{array}{l}\text { Redness (baseline; score }=0 \text { ) } \\
\text { Redness (baseline; score }=1 \text { ) }\end{array}$ & $\begin{array}{l}0(0 \%) \\
9(17 \%)\end{array}$ & $\begin{array}{l}0(0 \%) \\
15(29 \%)\end{array}$ & 0.32 \\
\hline Redness (baseline; score $=2$ ) & $19(37 \%)$ & $14(27 \%)$ & \\
\hline Redness (baseline; score $=3$ ) & $24(46 \%)$ & $23(44 \%)$ & \\
\hline $\begin{array}{l}\text { Redness (follow-up; score }=0 \text { ) } \\
\text { Redness (follow-up; score }=1 \text { ) }\end{array}$ & $\begin{array}{l}0(0 \%) \\
17(33 \%)\end{array}$ & $\begin{array}{l}21(40 \%) \\
18(35 \%)\end{array}$ & $<0.01$ \\
\hline Redness (follow-up; score $=2$ ) & $15(29 \%)$ & $13(25 \%)$ & \\
\hline Redness (follow-up; score $=3$ ) & $20(38 \%)$ & $0(0 \%)$ & \\
\hline $\begin{array}{l}\text { Edema (baseline; score }=0 \text { ) } \\
\text { Edema (baseline; score }=1 \text { ) }\end{array}$ & $\begin{array}{l}0(0 \%) \\
12(23 \%)\end{array}$ & $\begin{array}{l}0(0 \%) \\
12(23 \%)\end{array}$ & 0.189 \\
\hline Edema (baseline; score $=2$ ) & $28(54 \%)$ & $20(38 \%)$ & \\
\hline Edema (baseline; score $=3$ ) & $12(23 \%)$ & $20(38 \%)$ & \\
\hline $\begin{array}{l}\text { Edema (follow-up; score }=0 \text { ) } \\
\text { Edema (follow-up; score }=1 \text { ) }\end{array}$ & $\begin{array}{l}0(0 \%) \\
20(38 \%)\end{array}$ & $\begin{array}{l}23(44 \%) \\
23(44 \%)\end{array}$ & $<0.01$ \\
\hline Edema (follow-up; score = 2) & $21(40 \%)$ & $6(12 \%)$ & \\
\hline Edema (follow-up; score = 3) & $11(21 \%)$ & $0(0 \%)$ & \\
\hline
\end{tabular}

extensively used in the orthopedic and rheumatology clinics [20, 21]. CRP is increased during the acutephase response while it returns to physiological values following tissue recovery and functional restoration [22]. Therefore, in this study, CRP was used to allow costeffective monitoring of the pharmacological treatment efficacy.

Statistical analysis Patients' age is presented as mean $\pm \mathrm{SEM}$; VAS, WOMAC, and CRP data are reported as median [interquartile range (IQR)]; edema and redness data are presented as frequencies (percentages) (Table 1). Comparison of age between groups was performed using Student's $t$ test. Comparison of posttreatment percentage variation from baseline for VAS, WOMAC, and CRP data was performed using Wilcoxon's signed-rank test. Comparison of posttreatment percentage variation for edema and redness data from baseline was performed using Pearson's chisquare test. A $p$ value $<0.05$ was considered significant. All statistical analyses were conducted using $\mathrm{R}$ software [23]. 


\section{Results}

Table 1 shows baseline and 10-day post-treatment patients' characteristics. At 10-day follow-up, a greater reduction in VAS (Fig. 1a) and WOMAC pain (Fig. 1b) scores from baseline was observed in patients treated with Betesil (55.44 \pm $1.28 \%$ and $52.4 \pm 2.31 \%$, respectively), when compared with diclofenac sodium cream $(13.89 \pm 0.8 \%$ and $12.35 \pm 1.26 \%$, respectively; all $p<0.01$ ). A greater reduction in WOMAC functional limitation (Fig. 1c) and stiffness (Fig. 1d) scores from baseline was observed in patients treated with Betesil $(44.79 \pm 2.33 \%$ and $50.03 \pm 3.08 \%$, respectively), when compared with diclofenac sodium cream $(9.62 \pm 0.79 \%$ and $14.94 \pm 2.26 \%$, respectively; all $p<0.01)$. A similar trend was observed for redness (Fig. 2a) and edema (Fig. 2b) scores in patients treated with Betesil, when compared with diclofenac sodium cream (all $p<0.01$ ). A greater reduction in CRP levels (Fig. 3) from baseline was also observed in patients treated with Betesil (46.54 $\pm 3.52 \%)$, when compared with diclofenac sodium cream $(14.42 \pm 1.25 \% ; p<0.01)$. During the study, diclofenac sodium was used by 15 patients; 10 patients took two capsules a day for 2 days and then a capsule
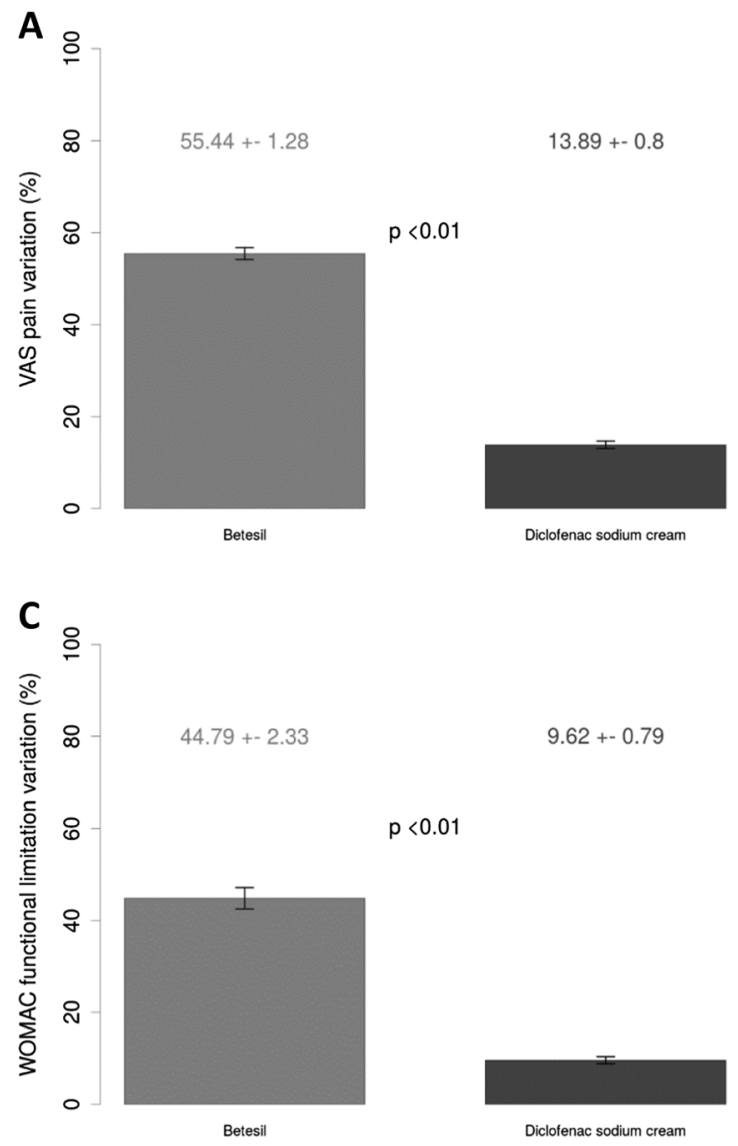

Fig. 1 Betesil improves arthritis and osteoarthritis pain. Percentage variation in VAS pain (a), WOMAC pain (b), WOMAC functional limitation (c), and WOMAC stiffness (d) scores from baseline at 10-day a day for 2 days during the first 4 days of plaster application. A total of five patients took a capsule a day for 2 days, during the first 2 days of plaster application. Only one patient reported a skin rash 4 hours after the first application of the plaster. The rash was thought to be an allergic reaction and resolved without medication after 5 hours. This patient continued applying the plaster the following days and completed the study.

\section{Discussion}

To the best of our knowledge, this is the first demonstration of the clinical effectiveness and safety of a BMV medicated plaster for treatment of symptomatic joint arthritis and OA. Scientific interest in transdermal drug delivery systems has significantly increased in the last two decades because this approach is considered a valid therapeutic alternative to oral and more invasive strategies. The advantages of transdermal drug delivery include better patient compliance, control over input kinetics, and a lower incidence of gastrointestinal-related side effects [24], as observed in this study. Furthermore, it avoids the first-pass hepatic metabolism and plasma bioavailability
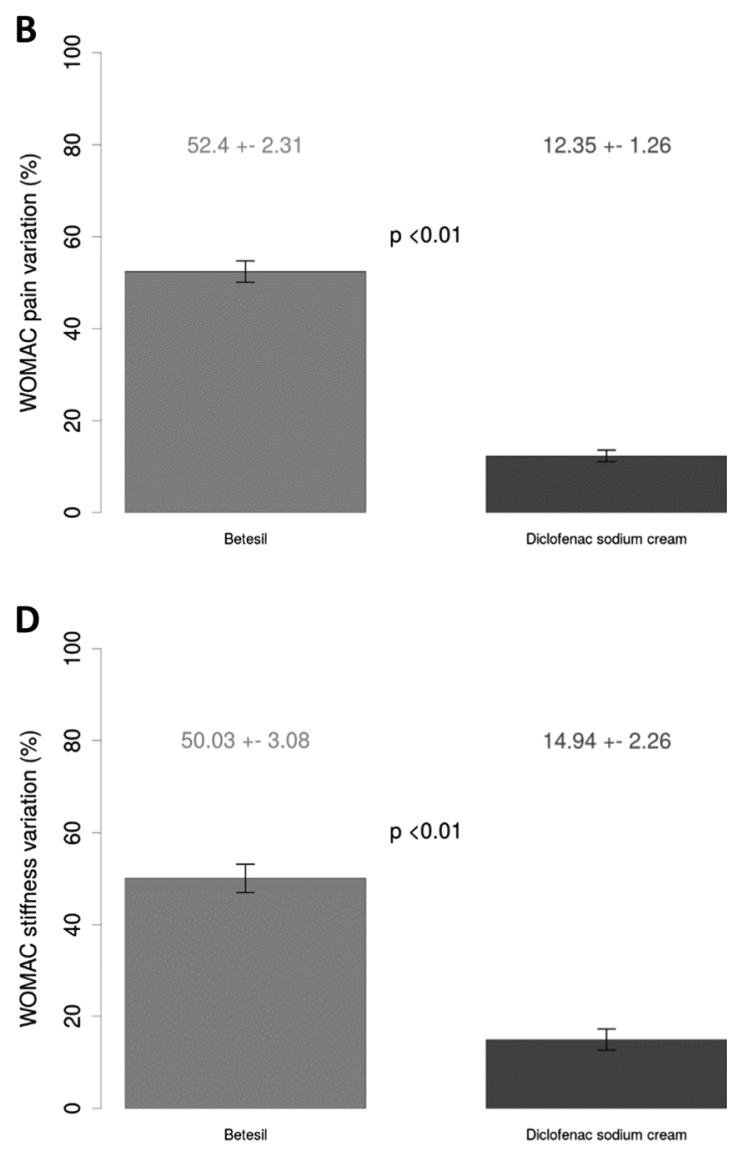

follow-up following Betesil and diclofenac sodium cream treatment in patients affected by arthritis and osteoarthritis affecting different joints 
A

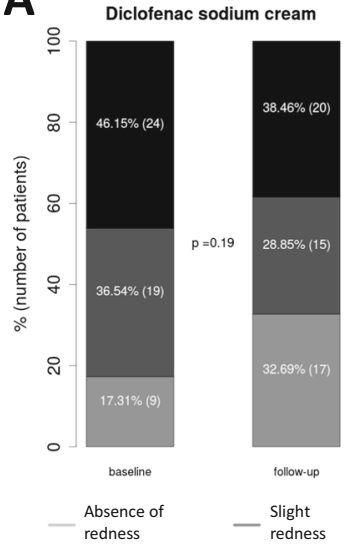

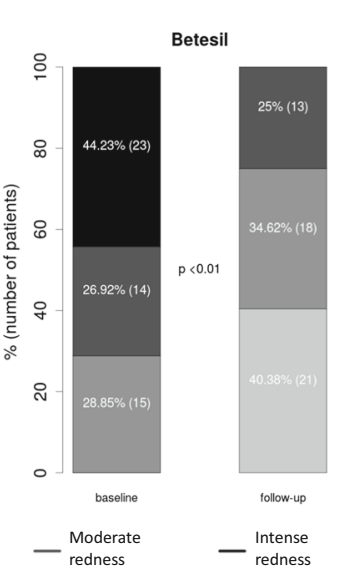

B

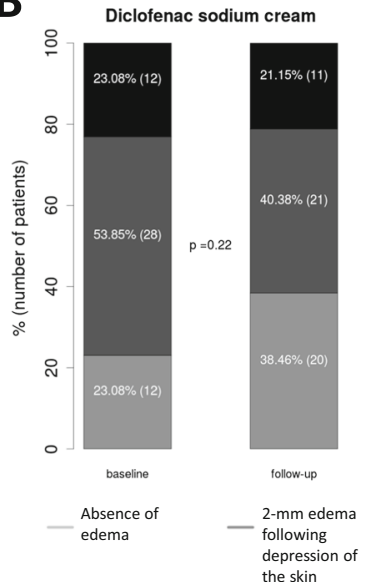

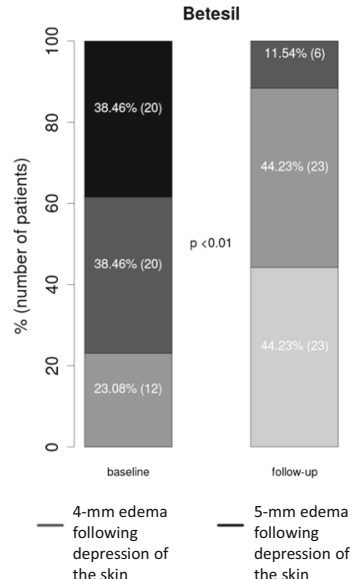

Fig. 2 Betesil improves redness and edema scores in arthritis and osteoarthritis patients. Percentage variation in redness (a) and edema (b) from baseline at 10-day follow-up following Betesil and diclofenac sodium cream treatment in patients affected by arthritis and osteoarthritis affecting different joints

fluctuations usually observed with oral administration [25]. In conclusion, the present study shows that Betesil is well tolerated and displays superior efficacy in reducing pain and functional disability and ameliorating CRP levels, redness, and edema, when compared with diclofenac sodium cream, in patients affected by arthritis and OA. The improvement in CRP levels supports tissue recovery and functional restoration [22] allowing us to monitor the therapeutic efficacy of Betesil and further supporting its use as a local pharmacological therapy for arthritis pain and functional disability.

Authors' contributions TI, MFM, CL, AM, and BP conceived the study, drafted the manuscript, participated in the design of the study and data collection, and performed the

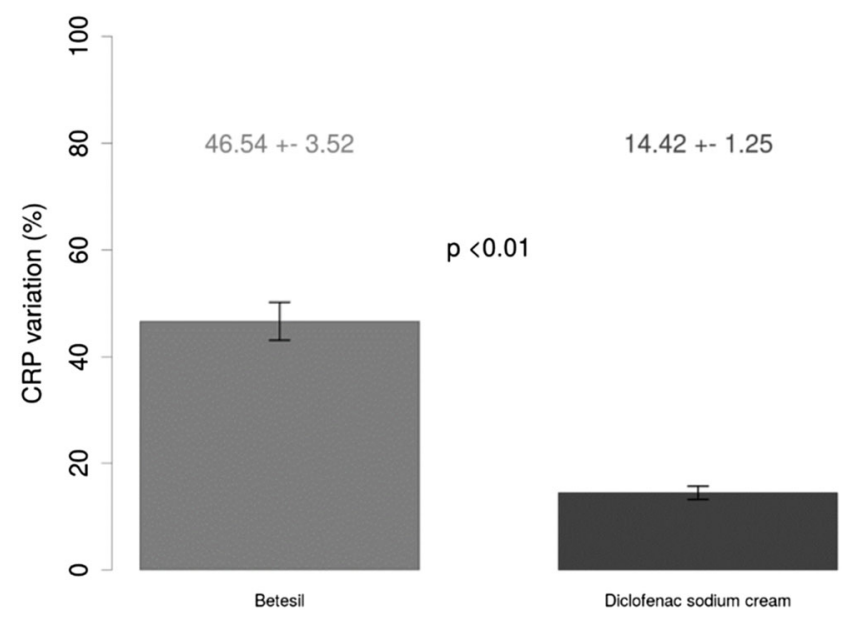

Fig. 3 Betesil improves CRP in arthritis and osteoarthritis patients. Percentage variation in CRP from baseline at 10-day follow-up following Betesil and diclofenac sodium cream treatment in patients affected by arthritis and osteoarthritis affecting different joints statistical analysis. BP coordinated the study. All authors read and approved the final manuscript.

Compliance with ethical standards Written informed consent was obtained from the patients for publication of their details in this manuscript. The consent form is held by the authors in the patients' clinical notes and is available for review by the Editor-in-Chief. This study was performed at the Poliambulatorio del Secondo Parere clinic (Modena, Italy). The Institutional Review Board at Poliambulatorio del Secondo Parere (Modena, Italy) approved this study.

Conflict of interest The authors declare that they have no conflict of interest.

Funding statement This article was not supported by any grants.

Open Access This article is distributed under the terms of the Creative Commons Attribution 4.0 International License (http:// creativecommons.org/licenses/by/4.0/), which permits unrestricted use,

distribution, and reproduction in any medium, provided you give appropriate credit to the original author(s) and the source, provide a link to the Creative Commons license, and indicate if changes were made.

\section{References}

1. Blalock D, Miller A, Tilley M, Wang J. Joint instability and osteoarthritis. Clin Med Insights Arthritis Musculoskelet Disord. 2015;8: $15-23$.

2. Snibbe JC, Gambardella RA. Treatment options for osteoarthritis. Orthopedics. 2005;28:s215-20.

3. Fary RE, Carroll GJ, Briffa TG, Gupta R, Briffa NK. The effectiveness of pulsed electrical stimulation (E-PES) in the management of osteoarthritis of the knee: a protocol for a randomised controlled trial. BMC Musculoskelet Disord. 2008;9:18.

4. Bennell KL, Hunter DJ, Hinman RS. Management of osteoarthritis of the knee. BMJ. 2012;345:e4934. 
5. Iannitti Tet al. Bisphosphonates: focus on inflammation and bone loss. Am J Ther. 2012;19:228-46.

6. Iannitti T, Fistetto G, Esposito A, Rottigni V, Palmieri B. Pulsed electromagnetic field therapy for management of osteoarthritisrelated pain, stiffness and physical function: clinical experience in the elderly. Clin Interv Aging. 2013;8:1289-93.

7. Palmieri B, Rottigni V, Iannitti T. Preliminary study of highly crosslinked hyaluronic acid-based combination therapy for management of knee osteoarthritis-related pain. Drug design, development and therapy. 2013;7:7-12.

8. Iannitti T, Lodi D, Palmieri B. Intra-articular injections for the treatment of osteoarthritis: focus on the clinical use of hyaluronic acid. Drugs R D. 2011;11:13-27.

9. Iannitti T, Rottigni V, Palmieri B. A pilot study to compare two different hyaluronic acid compounds for treatment of knee osteoarthritis. Int J Immunopathol Pharmacol. 2012;25:1093-8.

10. Iannitti T, Elhensheri M, Bingol AO, Palmieri B. Preliminary histopathological study of intra-articular injection of a novel highly cross-linked hyaluronic acid in a rabbit model of knee osteoarthritis. J Mol Histol. 2013;44:191-201.

11. Li P et al. Hylan G-F 20 maintains cartilage integrity and decreases osteophyte formation in osteoarthritis through both anabolic and anti-catabolic mechanisms. Osteoarthritis Cartilage. 2012;20(11): 1336-46.

12. Ringdahl E, Pandit S. Treatment of knee osteoarthritis. Am Fam Physician. 2011;83:1287-92.

13. Stanos SP, Galluzzi KE. Topical therapies in the management of chronic pain. Postgrad Med. 2013;125:25-33.

14. Nelson AE, Allen KD, Golightly YM, Goode AP, Jordan JM. A systematic review of recommendations and guidelines for the management of osteoarthritis: the chronic osteoarthritis management initiative of the U.S. bone and joint initiative. Semin Arthritis Rheum. 2014;43:701-12. doi:S0049-0172(13)00258-8

15. Iannitti T, Rottigni V, Palmieri B. Corticosteroid transdermal delivery to target swelling, edema and inflammation following facial rejuvenation procedures. Drug Des Devel Ther. 2013;7:1035-41.

16. Evans RM. The steroid and thyroid hormone receptor superfamily. Science. 1988;240:889-95.

17. Mina $\mathrm{R}$ et al. Effectiveness of dexamethasone iontophoresis for temporomandibular joint involvement in juvenile idiopathic arthritis. Arthritis Care Res (Hoboken). 2011;63:1511-6.

18. Jain R et al. Evaluation of transdermal steroids for trapeziometacarpal arthritis. J Hand Surg Am. 2010;35:921-7.

19. Fogari R. Semeiotica medica e metodologia clinica. Piccin Nuova Libraria. Padova (1999). ISBN 88-299-1506-8.

20. Foglar C, Lindsey RW. C-reactive protein in orthopedics. Orthopedics. 1998;21(6):687-91; quiz 692-3

21. van Leeuwen MA, van Rijswijk MH. Acute phase proteins in the monitoring of inflammatory disorders. Baillieres Clin Rheumatol. 1994;8:531-52.

22. Tarik MH, David HK. C-reactive protein and erythrocyte sedimentation rate in orthopaedics. The University of Pennsylvania Orthopaedic Journal. 2002;15:13-16.

23. Team RC. R: a language and environment for statistical computing. R Foundation for Statistical Computing, Vienna, Austria. URL: https://www.R-project.org/. (2015).

24. N'Da DD. Prodrug strategies for enhancing the percutaneous absorption of drugs. Molecules. 2014;19:20780-807.

25. L'Hermite M, Simoncini T, Fuller S, Genazzani AR. Could transdermal estradiol + progesterone be a safer postmenopausal HRT? A review. Maturitas. 2008;60:185-201. 